\title{
Behavioral plasticity mitigates risk across environments and predators during anuran metamorphosis
}

\author{
Justin C. Touchon · Randall R. Jiménez • \\ Shane H. Abinette $\cdot$ James R. Vonesh $\cdot$ \\ Karen M. Warkentin
}

Received: 22 February 2013 / Accepted: 10 June 2013

(C) Springer-Verlag Berlin Heidelberg 2013

\begin{abstract}
Most animals metamorphose, changing morphology, physiology, behavior and ecological interactions. Size- and habitat-dependent mortality risk is thought to affect the evolution and plastic expression of metamorphic timing, and high predation during the morphological transition is posited as a critical selective force shaping complex life cycles. Nonetheless, empirical data on how risk changes across metamorphosis and stage-specific habitats, or how that varies with size, are rare. We examined predator-prey interactions of red-eyed treefrogs, Agalychnis callidryas, with an aquatic predator (giant water bug, Belostoma) and a semi-terrestrial predator (fishing spider, Thaumasia) across metamorphosis. We manipulated tadpole density to generate variation in metamorph size and conducted predation trials at multiple developmental stages. We quantified how frog behavior (activity) changes across metamorphic development, habitats, and predator presence or absence. In aquatic trials with water bugs, frog mortality increased with forelimb emergence, as hypothesized.
\end{abstract}

Communicated by Steven Kohler.

J. C. Touchon $\cdot$ K. M. Warkentin $(\bowtie)$

Department of Biology, Boston University, 5 Cummington Mall,

Boston, MA 02215, USA

e-mail: kwarken@bu.edu

J. C. Touchon · K. M. Warkentin

Smithsonian Tropical Research Institute, Apartado Postal

0843-03092, Panama, Republic of Panama

R. R. Jiménez

Departamento de Biología Tropical, Universidad Nacional, Heredia 40101, Costa Rica

S. H. Abinette · J. R. Vonesh

Department of Biology, Virginia Commonwealth University, 100 West Cary Street, Richmond, VA 23284, USA
In semi-terrestrial trials, contrary to predictions, predation by spiders increased, not decreased, with tail resorption. In neither case did frog size affect mortality. Frogs reduced activity upon forelimb emergence in the water, and further with emergence into air, then increased activity with tail resorption. Longer-tailed metamorphs were captured more often in spider attacks, but attacked less, as most attacks followed prey movements. Metamorphs behaviorally compensated for poor escape performance more effectively on land than in water, thus emergence timing may critically affect mortality. The developmental timing of the ecological transition between environments that select for different larval and juvenile phenotypes is an important, neglected variable in studies of complex life cycles.

Keywords Complex life cycle $\cdot$ Escape performance · Phenotypic plasticity $\cdot$ Predation $\cdot$ Trade-off

\section{Introduction}

Most animals have complex life cycles that include multiple ecologically and often morphologically and physiologically distinct life stages (Moran 1994; Wilbur 1980). Complex life cycles exist, at least in part, because selection changes with body size; how life cycles are partitioned across stages reflects how costs and benefits in each stage scale with size (Werner 1986, 1988; Werner and Gilliam 1984). Phenotypic differences between stages (e.g., larval vs. adult morphology, physiology, and behavior) reflect differences in selection on different stages. With increasing stage-specific adaptation, the transition between stages becomes more dramatic, so the nature of metamorphosis varies; for instance, the phenotypic change at metamorphosis increases from fishes to urodeles to anurans (Laudet 
2011; Szarski 1957). Metamorphosis has long been the focus of intense scientific interest and research. Nonetheless, to our knowledge, there has been no direct assessment of how the two key factors, size and developmental phenotype (stage within metamorphosis), jointly affect survival in the habitats of both life stages.

Research in evolutionary ecology has largely viewed metamorphosis in one of two conceptual frameworks-as a switch point between life stages or as a dangerous period during development. A substantial body of theory and empirical research analyzes metamorphosis as the point of separation between two life stages, focusing on how variation in selection on each stage shapes the size at and timing of the transition (Werner 1986; Werner and Gilliam 1984). Empirical work has amply demonstrated the plasticity of these traits. However, some initial hypotheses, particularly the prediction that higher predation of larvae would lead to metamorphosis earlier and smaller, have turned out to be too simple (Benard 2004; Relyea 2007). For instance, behavioral responses of larvae to predators can slow growth and delay metamorphosis. An integrative perspective incorporating multiple plastic responses of prey to predator cues, along with direct and indirect effects of predators, has better explanatory power (Relyea 2007). This research does not, however, directly address effects of the developmental changes that occur during metamorphosis on fitness in either environment.

A separate body of work treats metamorphosis as a distinct period, focusing on its dangers. Animals in transition between two distinct adaptive phenotypes may necessarily pass through a period of low performance, and consequently high risk (Arnold and Wassersug 1978; Wassersug and Sperry 1977). High mortality of transforming animals should select to reduce the period of functional incompetence and accelerate development through metamorphosis (Downie et al. 2004; Landberg and Azizi 2009; Wassersug and Sperry 1977). Poor performance during transformation could increase mortality in either habitat, although not necessarily equally. In addition, animals entering a new habitat might suffer high mortality because they are unfamiliar with the habitat, have to traverse a dangerous area to reach it, or have not yet found an appropriate new refuge or territory. High mortality associated with metamorphosis has been documented in reef fishes (Almany and Webster 2006), benthic marine invertebrates (Gosselin and Qian 1997), insects (Corbet 1962), and amphibians (Arnold and Wassersug 1978; Crump 1984; Wassersug and Sperry 1977). However, the relative importance of poor performance and entry into a novel environment are not always clear and likely vary among taxa and contexts.

Considering how much mortality can occur during metamorphosis, and thus the potential for strong selection, it is surprising how little research has addressed how factors such as size at or timing of metamorphosis, or the behavior of metamorphs, might modulate risk during this critical period (but see Vail and McCormick 2011). However, the view of metamorphosis as an inevitably dangerous time, where the only solution is to shorten it, is probably too simple (Downie et al. 2004; Drummond and García 1989; Landberg and Azizi 2009). Like embryogenesis, metamorphosis is a period when multiple morphological and physiological traits are changing rapidly (Downie et al. 2004; Warkentin 2011a). These traits contribute to multiple aspects of performance that vary in their importance for fitness both across habitats and with conditions in each habitat. During more dramatic metamorphoses, as during embryogenesis, animals subsist on stored reserves (Orlofske and Hopkins 2009), so selection is focused on survival and development, not growth. Thus, as with hatching (Warkentin 2011a, b), when biotic or abiotic risks differ between habitats, animals should time their habitat transition within metamorphosis in response to environmental conditions. To understand the optimal timing of the habitat switch, we need to know how developmental changes affect mortality risk in each habitat, as well as how this relationship may be modulated by other factors including size and behavior (De Vito 2003; Vail and McCormick 2011). This information will also improve our understanding of how selection may act on the timing of, size at, or speed of metamorphosis.

Anurans are important models for understanding metamorphosis and the focus of a large body of ecological, developmental, and evolutionary research on complex life cycles (Relyea 2007; Shi 2000; Szarski 1957; Werner 1986). Predation has been proposed as the source of selection for rapid metamorphosis (Szarski 1957), and ontogenetic studies of locomotor performance at metamorphosis are typically framed in this context (Brown and Taylor 1995; Huey 1980; Walsh et al. 2008a). Predation is also considered a major factor affecting the timing of and size at metamorphosis, across taxa (Benard 2004; Relyea 2007; Werner 1986; Werner and Gilliam 1984). Nonetheless, surprisingly little is known about how predation risk changes across metamorphosis for any taxa, even the well-studied anurans. To our knowledge, only one study, using a single predator, has experimentally assessed the ontogeny of risk to metamorphosing frogs both in water and on land (Wassersug and Sperry 1977). An observational field study found anurans with forelimbs and tails to be disproportionately abundant in garter snake guts (Arnold and Wassersug 1978), but this might simply be due to prey habitat use (Drummond and García 1989). Two studies experimentally assessed how forelimb emergence affects aquatic predation: it increased with 3 different predators (Crump 1984, 1986; see also Calsbeek and Kuchta 2011). In addition, a few studies have documented that anurans alter behavior 
or development at metamorphosis in response to predators, emerging from the water more synchronously (De Vito 2003; De Vito et al. 1998), changing the developmental timing of emergence from the water (Vonesh and Warkentin 2006), or shortening the period from forelimb emergence through tail resorption (Walsh et al. 2008b).

Here, we assess how predation risk varies across metamorphosis, habitat, and size. Our general hypotheses were: (1) that risk is higher during metamorphosis than in earlier or later stages, as expected in the transition between distinct adaptive phenotypes; (2) that risk decreases with increasing prey size, as is common in predator-prey interactions; and (3) that these patterns pertain in the habitats of both larval and juvenile stages. We compared the survival of morphologically transitional anurans of different sizes to less-developed animals in their larval habitat and to more-developed animals in their juvenile habitat, using predators common in each habitat. We conducted predation trials to measure patterns of mortality, assessed behavioral responses to predator cues in both habitats, and made focal observations of predator-prey interactions to elucidate underlying mechanisms. Following accepted assumptions, we hypothesized that anuran vulnerability peaks at metamorphic climax, when forelimbs emerge on animals with long tails. Thus, our specific predictions were that in the water the risk of predation increases with forelimb emergence, while out of water the risk of predation decreases with tail resorption, and in both contexts, smaller prey suffer higher predation.

\section{Materials and methods}

\section{Study system}

We worked in Gamboa, Panama, at the Smithsonian Tropical Research Institute. Our study species were red-eyed treefrogs (Agalychnis callidryas), giant water bugs (Belostoma cf. porteri.), and fishing spiders (Thaumasia sp.). All are abundant in local ponds. Red-eyed treefrogs are widespread in Neotropical wet forests from the Yucatan to northern Colombia (Duellman 2001). Belostomatids are common aquatic predators of anuran larvae that also feed on metamorphs and breeding adults at ponds (Gascon 1992; Hirai and Hidaka 2002; Jara and Perroti 2010; Toledo 2003). Spiders prey on anurans on land and at the water surface (Jara 2008; Menin et al. 2005; Moore and Townsend 1998), and are considered particularly important predators of emerging metamorphs (Donnelly and Guyer 1994). Cues from both predators affect the metamorphic timing of A. callidryas: metamorphs emerge from the water smaller and earlier in development in response to Belostoma and larvae delay metamorphosis to emerge at a larger size in response to Thaumasia (Vonesh and Warkentin 2006). Animals exposed to cues from both predators respond to the Belostoma, emerging at a relatively small size and with longer tails (Vonesh and Warkentin 2006). These responses suggest that transitional morphology may impair survival more with water bugs than with spiders and that larger size may be beneficial for survival with spiders (Vonesh 2005).

All experiments were conducted in a partially shaded field at the forest edge beside the Experimental Pond in Gamboa, using a mixture of captured rainwater and aged tap water. We conducted predation trials from July-November 2009, and made observations of behavior and predator-prey interactions from June-August 2011. Agalychnis were collected from Experimental Pond $\left(9^{\circ} 7^{\prime} 14.77^{\prime \prime} \mathrm{N}\right.$, $\left.79^{\circ} 42^{\prime} 12.03^{\prime \prime} \mathrm{W}\right)$. Spiders were collected from Experimental Pond, Ocelot Pond $\left(9^{\circ} 6^{\prime} 8.62^{\prime \prime} \mathrm{N}, 79^{\circ} 40^{\prime} 56.96^{\prime \prime} \mathrm{W}\right)$, and Kent's Marsh $\left(9^{\circ} 7^{\prime} 13.24^{\prime \prime} \mathrm{N}, 79^{\circ} 41^{\prime} 45.48^{\prime \prime} \mathrm{W}\right)$. Water bugs were collected from Quarry Pond $\left(9^{\circ} 7^{\prime} 22.25^{\prime \prime} \mathrm{N}, 79^{\circ}\right.$ $41^{\prime} 35.86^{\prime \prime} \mathrm{W}$ ). Predators and surviving A. callidryas were released at their collection site after trials.

Ontogeny of aquatic and terrestrial predation risk across metamorphosis

We exposed A. callidryas of different sizes and developmental stages to overnight predation trials in aquatic or semi-terrestrial habitats. Each trial exposed a single A. callidryas to a single water bug or spider, assessing survival to morning as a binary variable. Trials were conducted in 400-L, screen-covered, plastic mesocosms $(0.7 \mathrm{~m}$ diameter base, $0.9 \mathrm{~m}$ diameter mouth, $0.8 \mathrm{~m}$ high, with drain holes at $0.75 \mathrm{~m}$ height). For aquatic trials, mesocosms had a thin layer of leaf litter covering the bottom to provide habitat structure simulating an open pond. For semi-terrestrial trials, mesocosms had a second layer of screen $\sim 10 \mathrm{~cm}$ below the water surface and a continuous layer of floating vegetation (Salvinia sp. and Eichhornia sp.), simulating the water surface and pond-edge habitat where we find spiders preying on emerging metamorphs. Predator densities in mesocosms $\left(1.6 \mathrm{~m}^{-2}\right)$ were somewhat lower than observed at ponds for Thaumasia $\left(6 \pm 1.4 \mathrm{~m}^{-2}\right.$; Jara 2008) and higher than at ponds for Belostoma $\left(0.19-1.04 \mathrm{~m}^{-2}\right.$; Touchon and Vonesh, unpublished data). Agalychnis densities were also lower than in nature; this was necessary to test individual phenotypes.

All A. callidryas used in predation trials were collected as eggs and raised from hatching in $400-\mathrm{L}$, predator-free mesocosms at different densities (5, 25, and 50 tadpoles) to generate size variation. They were fed Sera micron powdered fish and tadpole food (Sera, Heinsberg, Germany). Animals were dipnetted from rearing tanks just before or after forelimb emergence (stage 41 or 42 ; Gosner 1960); 
we did not use metamorphs that had left the water themselves. For trials with water bugs, stage- 41 and $42 \mathrm{~A}$. callidryas were photographed for size measurements then immediately transferred to predation venues. For trials with spiders, metamorphs were used either immediately, at stage 42 with full-length tails, or after 1-4 days of tail resorption, (tail length range: 0-30.5 mm; stages 42-46). During tail resorption, metamorphs were held individually in plastic cups with perforated lids and shallow water. Metamorphs and predators were photographed with a ruler prior to trials, and measured using Image $(\mathrm{NIH})$.

We conducted 30 successful aquatic predation trials ( $n=15$ each at stages 41 and 42 ) with metamorphs ranging from 15.6 to $21.6 \mathrm{~mm}$ in snout-vent length $(19.0 \pm 1.6 \mathrm{~mm}$ SVL, mean \pm SD for all animal measurements). Aquatic trials used 19 different water bugs $(38.6 \pm 0.5 \mathrm{~mm}$ nosetip to end of abdomen). Five attempted stage- 42 trials were excluded because metamorphs climbed out of the water. We conducted 62 semi-terrestrial trials with metamorphs from 14.7 to $21.8 \mathrm{~mm} \mathrm{SVL}(18.6 \pm 1.6 \mathrm{~mm})$ and 40 different spiders $(15.2 \pm 3.9 \mathrm{~mm}$ cephalothorax + abdomen). The above trials included 15 successful trials with stage42 animals in each habitat (tail lengths $17.6-30.5 \mathrm{~mm}$, $25.4 \pm 0.85 \mathrm{~mm}$ ). The tail lengths of these metamorphs did not differ between trials with different predators (two-sample $t$ test, $t_{28}=1.2231, p=0.23$ ). For all trials, predator and prey were introduced simultaneously on opposite sides of mesocosms. Trials began at 1800-1830 hours and ran until 0800-0830 hours the following morning. Venues were left unused at least 2 days between trials. Predators that captured an A. callidryas were not used for at least 3 days before a subsequent trial. Predators were held individually in plastic cups with water between trials.

Ontogeny of aquatic activity and response to water bugs

To assess effects of water bug cues and forelimb emergence on A. callidryas behavior in the water, we conducted focal observations of 20 individuals at stage 41 and again at stage 42 . We collected stage- 41 tadpoles from the Experimental Pond and held them individually in water-filled plastic cups without predator cues for $<24 \mathrm{~h}$ before initial observations and then for another 1-4 days before final observations on the day of forelimb emergence. Individuals were randomly assigned to predator cue treatment (with or without, $n=10$ each). Observations were conducted in $60-\mathrm{L}$, round plastic tubs ( $0.4 \mathrm{~m}$ deep, $0.44 \mathrm{~m}$ diameter) covered with screen. Each tub initially contained a meshcovered cup; half of the cups housed a water bug and half were controls. Before observations, water bugs were each fed one A. callidryas tadpole (total length $22.3 \pm 1.9 \mathrm{~mm}$ ) to generate chemical cues of predation. Predators and cups were removed $1 \mathrm{~h}$ after feeding, then focal $A$. callidryas were placed individually in tubs and given $20 \mathrm{~min}$ to acclimate before observations. Observations were made in daylight, between 1300 and 1700 hours, to optimize underwater visibility. Although on land A. callidryas are nocturnally active, in the water they are active day and night, and water bugs prey on A. callidryas at all hours. We photographed predators before the experiment and A. callidryas immediately before both observation periods for size measurements (A. callidryas SVL: $18.40 \pm 1.61 \mathrm{~mm}$; water bugs: $40.59 \pm 1.38 \mathrm{~mm}, n=10)$. Anuran SVL did not change between observations $\left(F_{1,18}=3.15, p=0.10\right)$. Observations were made on a single $A$. callidryas at a time. Each animal was watched for ten 1-min focal observation periods, separated by 5 -min intervals, at each stage. We determined A. callidryas locations using a $10 \times 10$ grid of $25 \mathrm{~cm}^{2}$ squares drawn on the screen covering the tank and lines drawn in the tank at $7-\mathrm{cm}$ vertical intervals. We recorded the animal's position (3D grid section) at the start of observation periods and after each movement during observations, calculated distance moved by simple geometry, and averaged movement rates across the 10 observations of each animal at each stage.

\section{Ontogeny of terrestrial activity and response to spiders}

To assess behavior out of water and responses to spiders across tail resorption, we conducted nocturnal observations of individual $A$. callidryas metamorphs in 400-L mesocosms in the presence or absence of fishing spiders. Freeroaming spiders were used to provide cues in multiple sensory modalities. Eight mesocosms were set up with floating vegetation and a second screen as in semi-terrestrial predation trials; four were used for observations with spiders and four were predator-free. We used a total of 103 metamorphs $(18.8 \pm 1.2 \mathrm{~mm} \mathrm{SVL})$ and 12 spiders $(15.5 \pm 1.8 \mathrm{~mm})$. Stage-42 metamorphs were captured at night from the Experimental Pond and used immediately or kept for up to 5 days in cups, as above, to generate variation in tail length (range 0-31.0 mm). SVL and tail length were measured with calipers immediately before observations and metamorphs were randomly assigned to treatments. We placed metamorphs and spiders in their mesocosms, allowed $10 \mathrm{~min}$ for acclimation, then recorded metamorph locations every 20 min from 1930 to 2230 hours (10 observations per individual) using an 80-section radial grid drawn on the screens covering tanks. We used infrared-sensitive video cameras (Sony DCR-HC52) to avoid disturbing animals with visible light. We chose our sampling rate based on low movement frequency in continuously observed pilot trials and field observations; in $27 \%$ of the 20 -min checks, metamorphs had not moved. We estimated movement distances as the direct two-dimensional distance between the centers of grid sections occupied. We housed spiders in cups 
between trials, using each individual for observations every third night. Spiders were fed a small tadpole (total length $17.9 \pm 2.5 \mathrm{~mm}, n=17$ ) from the Experimental Pond in their cup after trials if their metamorph survived the night.

Effects of metamorph behavior and morphology on interactions with spiders

To further elucidate the nature of spider-metamorph interactions during predation attempts, we conducted 90-min focal observations in 3-L plastic containers $(19 \mathrm{~cm}$ diameter mouth, $15 \mathrm{~cm}$ base diameter, $11 \mathrm{~cm}$ height). The tubs were covered with screen, lined with mesh to facilitate climbing by spiders, and contained $\approx 1 \mathrm{~cm}$ of water and a continuous layer of floating Salvinia vegetation. We observed 62 metamorphs (SVL: $18.5 \pm 1.5 \mathrm{~mm}$ ) and 41 spiders $(12.7 \pm 1.7 \mathrm{~mm})$. Stage-42 metamorphs were collected from the Experimental Pond and used immediately or held in cups during tail resorption (tail length: $0-28.50 \mathrm{~mm}$ ). Spiders were housed in cups and used in one trial or two trials 6 days apart. They were fed every 3 days and left unfed for 2 days before trials; they received a small tadpole $(18.3 \pm 2.6 \mathrm{~mm})$ in their cup after trials if they did not eat in the trial. Before trials, we rinsed containers and vegetation to eliminate cues from prior trials, measured metamorph SVL and tail length with calipers, and photographed spiders for measurement. Observations were made between 1800 and 2100 hours, using red light to minimize disturbance to animals. The nocturnal behavior of $A$. callidryas is disrupted by white light but not by red light (Caldwell et al. 2010), and spiders appeared to behave normally under red light. For each trial, we placed a metamorph and a spider in a tub then observed their interactions continuously. We recorded all spider attacks on metamorphs and their outcomes. Attacks were distinguished by a spider initiating physical contact with a metamorph, i.e., jumping onto or grabbing it. Attacks were successful if the spider killed the metamorph or unsuccessful if the metamorph-by struggling, jumping, or submerging itself-escaped from contact with the spider. Metamorphs that escaped appeared uninjured; there was no evidence of envenomation and later mortality. For each attack, we recorded if the metamorph was moving or not immediately before the attack. We ran trials in batches, watching four tubs concurrently, and spider and metamorph activity levels were low enough for us to record all such behavioral interactions.

\section{Statistical Analysis}

All statistical analyses were conducted in R v.2.15.0 ( $\mathrm{R}$ Development Core Team 2012). To discriminate among competing models, we used Akaike's Information Criterion (AIC) to choose the best model. To assess predation risk across metamorphosis, we used generalized linear models (GLM) with an underlying binomial error distribution. The response variable was binary (metamorph survived or was eaten). Predictors were the presence/absence of emerged forelimbs, metamorph SVL, and their interaction for trials with water bugs and metamorph SVL, tail length, and their interaction for trials with spiders. We also compared the rates of predation by water bugs and spiders on stage- $42 \mathrm{~A}$. callidryas with forelimbs and long tails using a binomial GLM.

To assess activity of animals in the water, we used generalized linear mixed models (GLMM, lme4 package; Bates and Maechler 2009) with an underlying Gaussian distribution to test if distance moved by A. callidryas was affected by the presence/absence of forelimbs, the presence/absence of predator cues, and their interaction. "Individual" was included as a random intercept because we conducted observations on each animal twice. To assess activity out of the water, we used linear models (LM) to test if metamorph tail length, presence/absence of spiders, and their interaction affected distance moved. For behavioral observations of predator-prey interactions, we used an exact binomial test to test if spider attacks occurred more often on metamorphs that had just moved versus metamorphs that were still. We used Fisher's exact test to test if successful attacks were preceded by metamorph movement more often than were unsuccessful attacks. We used a GLM with underlying Poisson distribution to test if metamorph tail length predicted the number of attacks during the 90-min trial, excluding animals that were successfully captured which truncated observations. Lastly, we used a GLMM with binomial error distribution to test if the probability that an attack would be successful was predicted by metamorph SVL and tail length. The response variable was the outcome of each attack (successful or not) and we included individual as a random intercept in the model to account for non-independence of multiple attacks on the same metamorph. For GLMMs we estimated $p$ values of our predictors by using likelihood ratio tests of nested models (Zuur et al. 2009).

\section{Results}

Agalychnis callidryas with emerged forelimbs suffered greater predation by water bugs than did individuals without emerged forelimbs and prey size (SVL) had no significant effect (Fig. 1a; forelimbs: $\chi_{1,28}^{2}=16.0, p<0.00001$; SVL: $\left.\chi_{1,28}^{2}=0.7, p=0.40\right)$. Only 1 of $15(7 \%)$ of metamorphs with newly emerged forelimbs survived overnight in a mesocosm with a belostomatid, whereas $73 \%$ of tadpoles just prior to forelimb emergence survived (Fig. 1a). Both forelimb emergence and cues from water 

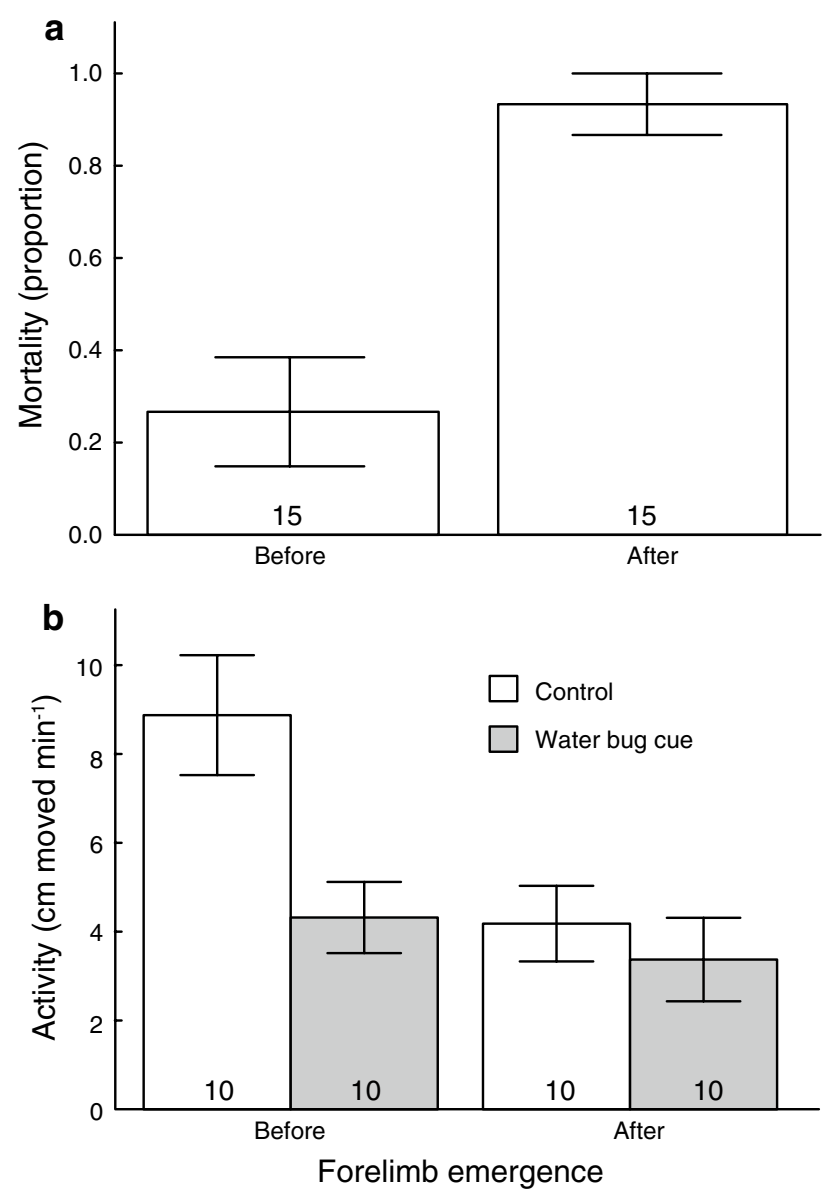

Fig. 1 Ontogenetic changes in aquatic predation and behavior of Agalychnis callidryas at metamorphosis. a Mortality in overnight trials with water bugs (Belostoma cf. porteri) and $\mathbf{b}$ activity level during focal observations of individuals with and without water bug cues, before and after forelimb emergence. Data are mean \pm SE. Sample sizes $(n)$ are indicated at the base of each bar

bug predation reduced A. callidryas' activity (Fig. 1b; forelimbs: $\chi^{2}=7.6, p=0.005$; predator cue: $\chi^{2}=6.6$, $p=0.01)$. There was also a non-significant trend toward a predator cue-forelimb emergence interaction (Fig. 1b; $\left.\chi^{2}=3.2, p=0.073\right)$. When exposed to predator cues, stage-41 tadpoles reduced movement by half while stage42 metamorphs decreased movement only slightly.

Contrary to our prediction, metamorphs with longer tails suffered less predation by spiders than did moredeveloped, shorter-tailed froglets. Tail length was the only important predictor of survival and metamorph SVL had no effect (Fig. 2a; tail length: $\chi_{1,59}^{2}=4.4 p=0.035$; SVL: $\chi_{1,59}^{2}=1.5, p=0.22$ ). The probability of predation increased $\sim 1.2 \%$ per $\mathrm{mm}$ of tail resorbed, i.e., by $\sim 35 \%$ with complete tail resorption (Fig. 2a). Metamorphs in large enclosures simulating pond edge habitat became more active with tail resorption but did not alter activity
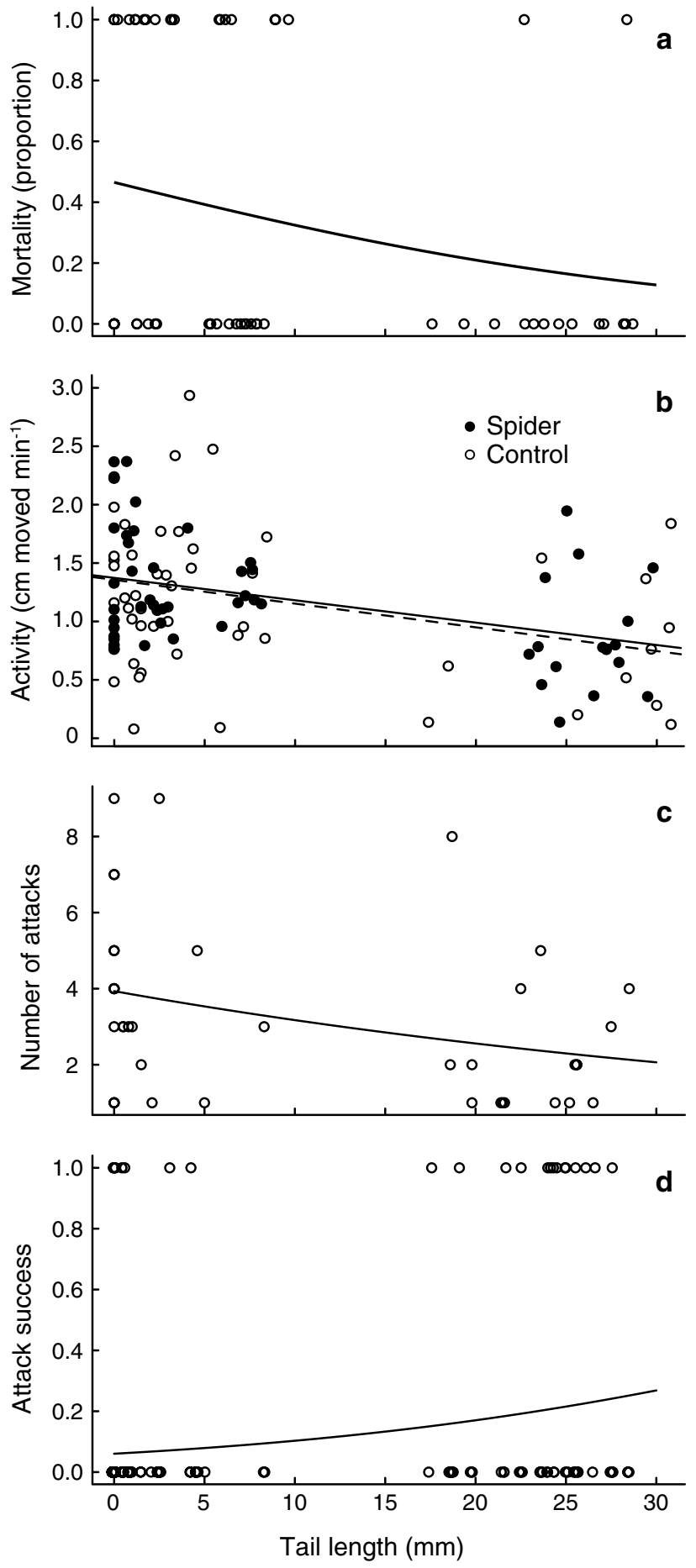

Fig. 2 Effect of tail resorption on interactions of Agalychnis callidryas metamorphs with fishing spiders, Thaumasia sp., in a semiterrestrial habitat. a Mortality in overnight trials, b activity level estimated from periodic nocturnal observations with and without spiders present, $\mathbf{c}$ number of attacks by spiders on individual metamorphs in 90-min focal observations, and $\mathbf{d}$ outcomes of attacks as successful captures or escapes (values at 1 and 0 , respectively). Lines are predicted fits from a a binomial generalized linear model, b linear regressions (dashed line control, solid spider), $\mathbf{c}$ a Poisson generalized linear model, and $\mathbf{d}$ a binomial generalized linear mixed model 
in response to spiders (Fig. 2b; tail length: $F_{1,99}=15.0$, $p=0.0002$; spider presence: $F_{1,99}=0.06, p=0.81$ ). Long-tailed metamorphs moved approximately half the distance of short-tailed metamorphs during our observations (Fig. 2b).

Predation on long-tailed metamorphs was higher in water than out of it $\left(\chi_{1,26}^{2}=20.2, p<0.0001\right)$. While water bugs consumed $93 \%$ of stage- 42 animals in overnight aquatic trials, spiders consumed only $13 \%$ in semiterrestrial trials. Neither metamorph SVL nor tail length predicted survival (SVL: $\chi_{1,26}^{2}=0.01, p=0.91$; tail: $\chi_{1,26}^{2}=0.08, p=0.77$ ).

In our observations of predator-prey interactions, we witnessed 167 attacks by spiders on metamorphs. Of those, 143 were immediately preceded by metamorph movement, so spiders were more likely to attack mobile than immobile metamorphs (exact binomial test, $p<0.00001$ ). Metamorphs with shorter tails were attacked about 1.6 times more often than were longer-tailed metamorphs (Fig. 2c; $\left.\chi_{1,37}^{2}=6.8, p=0.009\right)$. Attack outcome did not depend on whether metamorphs had just moved or were still when attacked (Fisher's exact test, $p=0.54$ ). However, attacks on metamorphs with longer tails were more likely to be successful, with success increasing $\sim 0.6 \%$ for every $\mathrm{mm}$ of tail remaining (Fig. 2d; tail length: $\chi^{2}=4.1, p=0.04$ ). Compared to fully metamorphosed froglets, the longesttailed metamorphs were approximately $20 \%$ more likely to be captured when they were attacked.

\section{Discussion}

Phylogenetically and ecologically diverse animals, from bivalves and barnacles (Gosselin and Qian 1997) to fishes (Almany and Webster 2006) and frogs, suffer a peak of mortality associated with metamorphosis and the move to their post-metamorphic habitat. Although details vary, both physiological stresses and predation are major challenges during this period. Prior research documented an ontogenetic peak in anuran vulnerability to predation at metamorphic climax (Wassersug and Sperry 1977). Our results indicate that developmental changes in behavior can complicate this picture. The relative importance of behavior and morphology can vary between habitats or predators, and realized predation rates may not match predictions from morphology. Moreover, the timing of habitat transitions can critically affect risk, and animals may adaptively alter the developmental stage at which they switch habitats in response to local risk conditions (Vonesh and Warkentin 2006). This possibility offers the opportunity to integrate two previously unconnected theoretical perspectives and bodies of research on complex life cycles and suggests important directions for future research. We first discuss our empirical findings, then consider their potential generality and implications for improving our integrative understanding of metamorphosis.

We found that, as predicted, belostomatid predation on A. callidryas in the water increased dramatically with forelimb emergence (Fig. 1a). Even though metamorphs reduced their activity by half with forelimb emergence, and further still in response to cues from water bugs (Fig. 1b; Vonesh and Warkentin 2006), this behavioral change was insufficient to protect them. In a semi-terrestrial environment, contrary to predictions from morphology, predation of metamorphs by spiders increased with tail resorption (Fig. 2a). Metamorphs were relatively inactive during the initial stages of tail resorption, regardless of spider presence (Fig. 2b). Although these long-tailed metamorphs were, as expected, poor at escaping from attacks, their relative immobility appeared to more than compensate for this by reducing the likelihood of attack; thus, long-tailed metamorphs were only $14 \%$ as likely to be killed out of the water as in it.

Across a 1.5-fold range of metamorph SVL (15-22 mm, equivalent to an almost threefold difference in mass at tail resorption; Touchon et al. 2013), size did not affect risk of predation by either water bugs or spiders. This result is in striking contrast to the common finding of size-dependent predation on tadpoles, including on A. callidryas tadpoles by Belostoma cf. porteri (McCoy et al. 2011) as well as size-dependent predation on fully metamorphosed froglets of another treefrog by spiders (Vonesh 2005). The insignificant effect of size on predation, with two different predators, during a period of rapid development highlights the importance of combining developmental and size-based functions to understand how risks vary across life cycles.

Realized predation rates depend on the combination of predator-prey encounter rates, the likelihood of attack given an encounter, and the likelihood of mortality given an attack, all of which may vary with traits of prey, predator, and habitat (Lima and Dill 1989). Anuran swimming performance can decline precipitously with forelimb emergence (Brown and Taylor 1995; Dudley et al. 1991; Huey 1980; Wassersug and Sperry 1977), although this is not always the case (Walsh et al. 2008b; Watkins 1997), and jumping performance can improve with tail resorption (Buckley et al. 2005; Wassersug and Sperry 1977). In A. callidryas, animals at metamorphic climax both swim and jump relatively poorly (T. Landberg and K.M. Warkentin, unpublished data), which could contribute to higher predation by Belostoma after forelimb emergence and lower escape success of long-tailed metamorphs in spider attacks. However, other traits, such as the number and size of graspable appendages, limb size and strength, and potentially struggling behavior, also change across these developmental stages and could affect attack outcomes. A pattern of 
mortality matched to changing locomotor performance is not sufficient to demonstrate that this is the critical determinant of either attack outcomes or predation rates (Drummond and García 1989; Ward-Fear et al. 2010).

Many prey, including anuran tadpoles (Richardson 2001), reduce activity in response to predator cues (Lima and Dill 1989), which can reduce both encounter rates and, for movement-cued predators, attack rates (Skelly 1994). In both aquatic and terrestrial environments, $A$. callidryas were less active at metamorphic climax than before or after, even without predator cues. Very low activity at metamorphic climax, and increasing activity with tail resorption, is consistent with focal observations of A. callidryas naturally emerging from a pond (Greene et al. 2011). This could reflect an innate defensive response by vulnerable developmental stages, shaped by a history of selection by predators. It could also be related to the physiological demands of the metamorphic process (Orlofske and Hopkins 2009; Shi 2000).

The fact that anurans do not feed while their mouthparts and digestive tracts are transforming eliminates foraging as a reason for movement. In water, however, anurans also move to breathe air (Feder 1984); A. callidryas surface frequently (Warkentin personal observation) and will die if denied access to the surface (Warkentin 1999). Although surfacing to breathe can elicit attacks by movement-cued predators (Bleckmann and Lotz 1987; Feder 1983; Moore and Townsend 1998), respiratory constraints likely set a minimum activity level for animals in the water. Indeed, at metamorphic climax, A. callidryas movement rate was about four times higher in the water, even with predation cues, than it was on land. Thus, if forelimb emergence compromises ability to escape from an aquatic predator, the best way for a metamorph to avoid predation may be to leave the water, as did $25 \%$ of such metamorphs in our overnight aquatic predation trials. We have been unable to do behavioral observations of predator-prey interactions of metamorphs in the water with Belostoma for just that reason; in smaller venues that generate observable interaction rates, metamorphs rapidly leave the water (Warkentin, personal observation; see Crump 1984 for similar observations on another frog species with 3 aquatic predators).

As with other fishing spiders that use cues from water surface waves (Bleckmann and Lotz 1987; Moore and Townsend 1998), Thaumasia attacks largely followed prey movement. Differences between the predators may be a factor, since belostomatids use both sit-and-wait and active foraging (Cloarec 1990). However, the ability of $A$. callidryas to more strongly reduce activity once on land likely contributes to their higher survival with spiders, compared to water bugs. The fact that metamorphs did not further reduce activity in response to spider cues contrasts with their response to water bugs, but is consistent with the change in activity, or lack thereof, of tadpoles in response to the same predators (Vonesh and Warkentin 2006). Nonetheless, A. callidryas does respond to spider cues, delaying metamorphosis to emerge larger and later (Vonesh and Warkentin 2006). We found no evidence that larger size aids survival with spiders, but their relatively short-tailed emergence phenotype in the absence of Belostoma cues, regardless of spider presence, would increase the odds of surviving spider attacks.

Our finding that forelimb emergence increases anuran vulnerability to aquatic predators is consistent with experimental studies on other species (Wassersug and Sperry 1977; Crump 1984, 1986) and is probably general across many anurans and predators. The apparent lack of modulation of this effect by even large differences in prey size has not, to our knowledge, been tested with other species, and this may vary. However, given the combination of reduced swimming performance and added graspable appendages with likely physiological limits on activity reduction, costs of forelimb emergence may often be large enough to overwhelm size effects in metamorph interactions with many aquatic predators.

Our finding that terrestrial predation increases with tail resorption is opposite the common assumption and the only other experimental test (Wassersug and Sperry 1977). How general might this be? Metamorph activity levels probably increase with tail resorption in other anurans, as energetic costs of both locomotion and bodily transformation lessen, as froglets become more physiologically suited to the terrestrial environment, and as froglets regain the ability to feed. The fact that prey activity commonly increases the likelihoods of encounter, detection, and attack by predators (Lima and Dill 1989), suggests that these components of the predation process may often increase with tail resorption. It may be that predation decreases with tail resorption only under the subset of conditions where escape performance is more important than likelihood of attack. The relative importance of attack and escape rates may also determine the extent to which size modulates ontogenetic patterns of predation on land; small size may often compromise escape performance but is unlikely to compromise the defensive value of immobility.

Towards a general framework for understanding flexible timing of metamorphosis

To understand how risk varies through complex life cycles, we will need to integrate the effects of growth, differentiation, and behavioral variation. These phenotypic changes occur on different time scales: growth is gradual; differentiation of morphology and physiology, although often slow, 

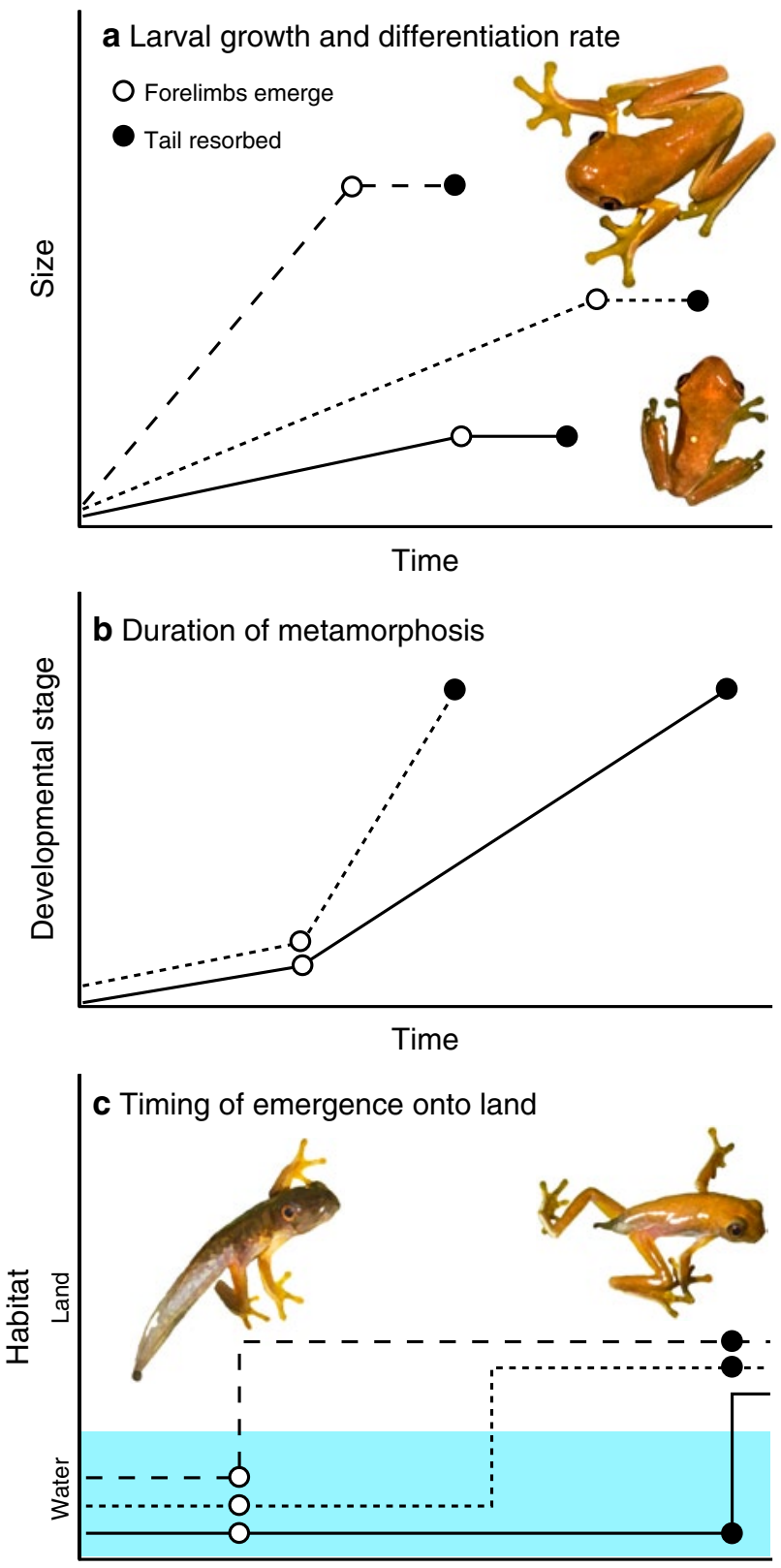

Developmental stage

Fig. 3 Three kinds of plasticity in anuran metamorphosis. a Larval growth and differentiation rates vary, affecting size at metamorphosis and time to metamorphosis. $\mathbf{b}$ The duration of metamorphosis varies (Downie et al. 2004; Walsh et al. 2008b), from forelimb emergence (Gosner stage 42, open circles) to tail resorption (stage 46, filled circles). c The point during development when animals move from water to land varies. Most ecological studies report either forelimb emergence or tail resorption, but not both, as measures of metamorphic timing, and do not report the phenotype at emergence onto land. Therefore, we know more about (a) than (b) or (c). The critical environmental conditions that select for different phenotypes in larval and juvenile stages (e.g., oxygen and water availability, types of predators and food resources) change at emergence from the water; thus the timing of and phenotype at emergence can strongly affect survival is dramatically rapid at metamorphosis; and behavior may change almost instantaneously in response to cues. For many animals during metamorphosis, developmental stage may be more relevant than size for many aspects of performance. For habitat changes associated with metamorphosis, the optimal stage for transition can vary with conditions in one or both habitats, and the immediate effects of differentiation on performance may be stronger or weaker in different habitats. When to switch habitats is fundamentally a behavioral decision, and can be influenced by both bodily condition and environmental cues (Elkin and Marshall 2007). For most animals, though, we know relatively little about either how behavior changes across stages of metamorphosis in their alternative habitats or the developmental timing of the habitat transition under different conditions.

Most ecological studies of plasticity in anuran metamorphic timing, including in response to larval predators, use either forelimb emergence or tail resorption as their endpoint (Walsh 2010; Fig. 3). Although they vary substantially, stage and tail length at the critical ecological transition, leaving the water, are very rarely reported (e.g., 0 of the 157 studies reviewed in Walsh 2010). Some A. callidryas climb onto land as soon as their forelimbs emerge, carrying a full-length, fully finned tail, while others remain in the water through the entire process of tail resorption (Touchon et al. 2013). We now know that the way risks change with development can vary across habitats and differ among predators, and that the ability of metamorphs to behaviorally compensate for morphological constraints on performance also varies across habitats. Thus, the developmental timing of emergence may be critically important for survival. It should be considered a response variable in ecological studies of metamorphosis (Fig. 3).

Across taxa, the developmental transition of metamorphosis is, in most cases, associated with a change in habitat and types of predators (Werner and Gilliam 1984). Both metamorphic morphology and entry into a new habitat at metamorphosis are thought to increase predation risk. Nonetheless, most studies have addressed risks in only one habitat, either pre- or post-metamorphic (Almany and Webster 2006; Calsbeek and Kuchta 2011; Corbet 1962; Crump 1984, 1986; Gosselin and Qian 1997; Jakob and Suhling 1999) or with a single predator across both habitats (Arnold and Wassersug 1978; Wassersug and Sperry 1977). Our comparison of changing predation risk at metamorphosis in two sequential habitats, to two different predators, is consistent with the hypothesis that transitional morphology can compromise escape ability in both environments. This is, however, only one of the factors that will determine predation rates at metamorphosis. Both constitutive 
developmental changes in behavior and plastic behavioral responses of metamorphs to predator cues or habitat characteristics may affect predation rates (De Vito 2003; Vail and McCormick 2011). Similarly, variation in metamorphic size, duration, or synchrony may alter predator-prey interactions (De Vito 2003; Downie et al. 2004; Walsh et al. 2008b).

The developmental timing of habitat switches at metamorphosis may also affect survival in abiotic selective contexts. For instance, marine invertebrates settling from pelagic to intertidal environments face dramatically different abiotic conditions, with more variable temperature, salinity, and oxygen, as well as risk of dehydration. Their stage of metamorphosis and physiological tolerance at the first low tide might be critically important in initial post-settlement mortality (Gosselin and Qian 1996). Amphibians' adaptation to terrestrial abiotic stresses likewise improves through metamorphosis, so less developed metamorphs may be at greater risk of dehydration and their emergence from the water may be more sensitive to weather than it is later in the metamorphic process.

Despite the long history of developmental research on metamorphosis, and the intensive ecological interest in metamorphosis as a switch point between stages in complex life cycles, we lack an integrative understanding of this critical ontogenetic period. Prior calls for integration across the ecology and developmental biology of metamorphosis focused on the regulation of larval period and its plasticity (Rose 2005). Others have pointed out the need to consider developmental plasticity in the process and duration of metamorphosis and its components, not just in its timing (Downie et al. 2004; Walsh et al. 2008b). We agree that these are important. We add here a call for research focused on the actual ecological transition between environments, each with its differing biotic and abiotic risks, that occurs sometime during the process of metamorphosis. Assessing risk trade-offs and behavior across this period of rapid development and determining how they vary with environmental conditions within and across habitats will be essential to build an integrative understanding of metamorphosis.

Acknowledgments We thank the Smithsonian Tropical Research Institute for use of their facilities and logistical support and the Autoridad Nacional del Ambiente de Panamá for permission to conduct this research (SE/A-32-09, SE/A-73-09, SC/A-13-11). We thank T. Landberg for assistance and M. Hughey and R. Wassersug for comments on the manuscript. This research was funded by the US National Science Foundation (DEB-0716923 to K.M.W. and DEB-0717220 to J.R.V.), Boston University, Virginia Commonwealth University, and the Smithsonian Tropical Research Institute.

\section{References}

Almany GR, Webster MS (2006) The predation gauntlet: early postsettlement mortality in reef fishes. Coral Reefs 25:19-22
Arnold SJ, Wassersug RJ (1978) Differential predation on metamorphic anurans by garter snakes (Thamnophis): social behavior as a possible defense. Ecology 59:1014-1022

Bates D, Maechler M (2009) lme4: Linear mixed-effects models using S4 classes. R package version 0.999375-32-2

Benard MF (2004) Predator-induced phenotypic plasticity in organisms with complex life histories. Annu Rev Ecol Syst 35:651-673

Bleckmann H, Lotz T (1987) Vertebrate-catching behaviour of the fishing spider Dolomedes triton (Araneae, Pisauridae). Anim Behav 35:641-651

Brown RM, Taylor DH (1995) Compensatory escape mode tradeoffs between swimming performance and maneuvering behavior through larval ontogeny of the wood frog, Rana sylvatica. Copeia 1995:1-7

Buckley CR, Michael SF, Irschick DJ (2005) Early hatching decreases jumping performance in a direct-developing frog, Eleutherodactylus coqui. Funct Ecol 19:67-72

Caldwell MS, Johnston GR, McDaniel JG, Warkentin KM (2010) Vibrational signaling in the agonistic interactions of red-eyed treefrogs. Curr Biol 20:1012-1017

Calsbeek R, Kuchta S (2011) Predator mediated selection and the impact of developmental stage on viability in wood frog tadpoles (Rana sylvatica). BMC Evol Biol 11:353

Cloarec A (1990) Factors influencing the choice of predatory tactics in a water bug, Diplonychus indicus Venk. \& Rao (Heteroptera: Belostomatidae). Anim Behav 40:262-271

Corbet PS (1962) A biology of dragonflies. Witherby, London

Crump ML (1984) Ontogenetic changes in vulnerability to predation in tadpoles of Hyla pseudopuma. Herpetologica 40:265-271

Crump ML (1986) Cannibalism by younger tadpoles: another hazard of metamorphosis. Copeia 1986:1007-1009

De Vito J (2003) Metamorphic synchrony and aggregation as antipredator responses in American toads. Oikos 103:75-80

De Vito J, Chivers DP, Kiesecker JM, Marco A, Wildy EL, Blaustein AR (1998) The effects of snake predation on metamorphosis of Western toads, Bufo boreas (Amphibia, Bufonidae). Ethology 104:185-193

Donnelly MA, Guyer C (1994) Patterns of reproduction and habitat use in an assemblage of Neotropical hylid frogs. Oecologia 98:291-302

Downie JR, Bryce R, Smith J (2004) Metamorphic duration: an understudied variable in frog life histories. Biol J Linn Soc 83:261-272

Drummond H, García CM (1989) Limitations of a generalist: a field comparison of foraging snakes. Behaviour 108:23-43

Dudley R, King VA, Wassersug RJ (1991) The implications of shape and metamorphosis for drag forces on a generalized pond tadpole (Rana catesbeiana). Copeia 1991:252-257

Duellman WE (2001) The hylid frogs of Middle America. Society for the Study of Amphibians and Reptiles, Ithaca

Elkin C, Marshall DJ (2007) Desperate larvae: influence of deferred costs and habitat requirements on habitat selection. Mar Ecol Prog Ser 335:143-153

Feder ME (1983) The relation of air breathing and locomotion to predation on tadpoles, Rana berlandieri, by turtles. Physiol Zool 56:522-531

Feder ME (1984) Consequences of aerial respiration for amphibian larvae. In: Seymour RS (ed) Respiration and metabolism of embryonic vertebrates. Junk, Dordecht, pp 71-85

Gascon C (1992) Aquatic predators and tadpole prey in central Amazonia: field data and experimental manipulations. Ecology 73:971-980

Gosner KL (1960) A simplified table for staging anuran embryos and larvae with notes on identification. Herpetologica 16:183-190

Gosselin LA, Qian P-Y (1996) Early post-settlement mortality of an intertidal barnacle: a critical period for survival. Mar Ecol Prog Ser 135:69-75 
Gosselin LA, Qian P-Y (1997) Juvenile mortality in benthic marine invertebrates. Mar Ecol Prog Ser 146:265-282

Greene RG, Noss CF, Landberg T, Vonesh JR, Warkentin KM (2011) Behavior and development of red-eyed treefrogs during metamorphosis. Integr Comp Biol 51:E195-E195

Hirai T, Hidaka K (2002) Anuran-dependent predation by the giant water bug, Lethocerus deyrollei (Hemiptera: Belostomatidae), in rice fields of Japan. Ecol Res 17:655-661

Huey RB (1980) Sprint velocity of tadpoles (Bufo boreas) through metamorphosis. Copeia 1980:537-540

Jakob C, Suhling F (1999) Risky times? Mortality during emergence in two species of dragonflies (Odonata: Gomphidae, Libellulidae). Aquat Insect 21:1-10

Jara FG (2008) Differential vulnerability of Physalaemus pustulosus tadpole size classes to predation by the water spider Thaumasia sp. (Physauridae). Amphibia-Reptilia 29:432-437

Jara FG, Perroti MG (2010) Risk of predation and behavioural response in three anuran species: influence of tadpole size and predator type. Hydrobiologica 644:313-324

Landberg T, Azizi E (2009) Ontogeny of escape swimming performance in the spotted salamander. Funct Ecol 24:576-587

Laudet V (2011) The origins and evolution of vertebrate metamorphosis. Curr Biol 21:R726-R737

Lima SL, Dill LM (1989) Behavioral decisions made under the risk of predation: a review and prospectus. Can J Zool 68:619-640

McCoy MW, Bolker BM, Warkentin KM, Vonesh JR (2011) Predicting predation through prey ontogeny using size-dependent functional response models. Am Nat 177:752-766

Menin M, de Jesus Rodrigues D, de Azevedo CS (2005) Predation on amphibians by spiders (Arachnida, Araneae) in the Neotropical region. Phyllomedusa 4:39-47

Moore MK, Townsend VR (1998) The interaction of temperature, dissolved oxygen and predation pressure in an aquatic predator-prey system. Oikos 81:329-336

Moran NA (1994) Adaptation and constraint in the complex life cycles of animals. Annu Rev Ecol Syst 25:573-600

Orlofske SA, Hopkins WA (2009) Energetics of metamorphic climax in the pickerel frog (Lithobates palustris). Comp Biochem Phys A 154:191-196

R Development Core Team (2012) R: a language and environment for statistical computing. R Foundation for Statistical Computing, Vienna, Austria

Relyea RA (2007) Getting out alive: how predators affect the decision to metamorphose. Oecologia 152:389-400

Richardson JML (2001) A comparative study of activity levels in larval anurans and response to the presence of different predators. Behav Ecol 12:51-58

Rose CS (2005) Integrating ecology and developmental biology to explain the timing of frog metamorphosis. Trends Ecol Evol 20:129-135

Shi Y-B (2000) Amphibian metamorphosis: from morphology to molecular biology. Wiley, New York

Skelly DK (1994) Activity level and the susceptibility of anuran larvae to predation. Anim Behav 47:465-468

Szarski H (1957) The origin of the larva and metamorphosis in Amphibia. Am Nat 91:283-301
Toledo LF (2003) Predation on seven South American anuran species by water bugs (Belostomatidae). Phyllomedusa 2:105-108

Touchon JC, McCoy MW, Vonesh JR, Warkentin KM (2013) Effects of plastic hatching timing carry over through metamorphosis in red-eyed treefrogs. Ecology 94:850-860

Vail AL, McCormick MI (2011) Metamorphosing reef fishes avoid predator scent when choosing a home. Biol Lett 7:921-924

Vonesh JR (2005) Sequential predator effects across three life stages of the African tree frog, Hyperolius spinigularis. Oecologia 143:280-290

Vonesh JR, Warkentin KM (2006) Opposite shifts in size at metamorphosis in response to larval and metamorph predators. Ecology $87: 556-562$

Walsh PT (2010) Anuran life history plasticty: variable practice in determining the end-point of larval development. Amphibia-Reptilia 31:157-167

Walsh PT, Downie JR, Monaghan P (2008a) Plasticity of the duration of metamorphosis in the African clawed toad. J Zool 274:143-149

Walsh PT, Downie JR, Monaghan P (2008b) Predation-induced plasticity in metamorphic duration in Xenopus laevis. Funct Ecol 22:699-705

Ward-Fear G, Brown GP, Shine R (2010) Factors affecting the vulnerability of cane toads (Bufo marinus) to predation by ants. Biol J Linn Soc 99:738-751

Warkentin KM (1999) Effects of hatching age on development and hatchling morphology in the red-eyed treefrog, Agalychnis callidryas. Biol J Linn Soc 68:443-470

Warkentin KM (2011a) Environmentally cued hatching across taxa: embryos respond to risk and opportunity. Integr Comp Biol 51:14-25

Warkentin KM (2011b) Plasticity of hatching in amphibians: evolution, trade-offs, cues and mechanisms. Integr Comp Biol 51:111-127

Wassersug RJ, Sperry DG (1977) The relationship of locomotion to differential predation on Pseudacris triseriata (Anura: Hylidae). Ecology 58:830-839

Watkins TB (1997) The effect of metamorphosis on the repeatability of maximal locomotor performance in the pacific tree frog Hyla regilla. J Exp Biol 200:2663-2668

Werner EE (1986) Amphibian metamorphosis: growth rate, predation risk, and the optimal size at transformation. Am Nat 128:319-341

Werner EE (1988) Size, scaling, and the evolution of complex life cycles. In: Ebenman B, Persson L (eds) Size-structured populations. Springer, Berlin, pp 60-81

Werner EE, Gilliam JF (1984) The ontogenetic niche and species interactions in size structured populations. Annu Rev Ecol Syst $15: 393-425$

Wilbur HM (1980) Complex life cycles. Annu Rev Ecol Syst 11:67-93

Zuur AF, Ieno EN, Walker NJ, Saveliev AA, Smith GM (2009) Mixed effects models and extensions in Ecology with R. Springer, New York 